\title{
Black hole mass estimates from soft X-ray spectra
}

\author{
Roberto Soria $^{\mathrm{a}, \mathrm{b}, *, 1}$, Zdenka Kuncic ${ }^{\mathrm{c}, 2}$ \\ ${ }^{\text {a } H a r v a r d-S m i t h s o n i a n}$ CfA, 60 Garden Street, Cambridge, MA 02138, USA \\ b Mullard Space Science Lab (UCL), Holmbury St. Mary, Surrey RH5 6NT, UK \\ ${ }^{c}$ School of Physics, University of Sydney, NSW 2006, Australia
}

Received 31 October 2006; accepted 2 March 2007

\begin{abstract}
In the absence of direct kinematic measurements, the mass of an accreting black hole is sometimes inferred from the X-ray spectral parameters of its accretion disk; specifically, from the temperature and normalization of a disk-blackbody model fit. Suitable corrections have to be introduced when the accretion rate approaches or exceeds the Eddington limit. We summarize phenomenological models that can explain the very high state, with apparently higher disk temperatures and lower inner-disk radii. Conversely, ultraluminous X-ray sources often contain cooler disks with large characteristic radii. We introduce another phenomenological model for this accretion state. We argue that a standard disk dominates the radiative output for radii larger than a characteristic transition radius $R_{\mathrm{c}} \sim \dot{m} \times R_{\mathrm{ISCO}}$, where $\dot{m}$ is the accretion rate in Eddington units and $R_{\mathrm{ISCO}}$ is the innermost stable orbit. For $R_{\mathrm{ISCO}}<R<R_{\mathrm{c}}$, most of the accretion power is released via non-thermal processes. We predict the location of such sources in a luminosity-temperature plot. We conclude that BHs with masses $\sim 50-100 M_{\odot}$ accreting at $\dot{m} \sim 10-20$ may explain the X-ray properties of many ULXs.
\end{abstract}

(C) 2007 COSPAR. Published by Elsevier Ltd. All rights reserved.

Keywords: Black holes; X-ray binaries; X-ray spectroscopy; X-ray sources; Infall; Accretion and accretion disks

\section{Standard disk in a thermal-dominant state}

Determining the masses of the accreting black holes (BHs) in ultraluminous X-ray sources (ULXs) is a key unsolved problem in X-ray astrophysics. In the absence of direct kinematic measurements of their mass functions, indirect methods based on X-ray spectral modelling have sometimes been used, by analogy with Galactic BH X-ray binaries, whose spectra can be approximated, in the "canonical" $0.3-10 \mathrm{keV}$ range, with a thermal component plus a power-law. The thermal component is consistent with disk-blackbody emission from an optically-thick disk, while the power-law is generally attributed to inverse-

\footnotetext{
Corresponding author. Address: Harvard-Smithsonian CfA, 60 Garden Street, Cambridge, MA 02138, USA.

E-mail address: rsoria@cfa.harvard.edu (R. Soria).

${ }^{1}$ Supported by a Marie Curie Outgoing International Fellowship.

${ }^{2}$ Supported by a University of Sydney Bridging Support Research Grant.
}

Compton scattering of softer disk photons off high-energy electrons. The peak temperature and luminosity of the thermal component are good indicators of the size of the $\mathrm{X}$-ray-emitting inner-disk region, which in turn is related to the $\mathrm{BH}$ mass.

In the standard disk-blackbody approximation (Makishima et al., 1986, 2000),

$$
\begin{aligned}
& L_{\text {disk }}=4 \pi \sigma\left(T_{\text {in }} / \kappa\right)^{4}\left(R_{\text {in }} / \xi\right)^{2} \equiv 4 \pi \sigma T_{\text {in }}^{4} r_{\text {in }}^{2}, \\
& R_{\text {in }}=R_{\text {ISCO }} \equiv 6 \alpha G M / c^{2},
\end{aligned}
$$

where $L_{\text {disk }}$ is the integrated (bolometric) disk luminosity; $T_{\text {in }}$ is the peak colour temperature, which can be directly inferred from X-ray spectral fitting ${ }^{3} ; T_{\text {in }} / \kappa$ is the peak effective temperature; $\kappa$ is the hardening factor $(1.5 \lesssim \kappa \lesssim 2.6)$; $R_{\text {in }}$ is the true inner-disk radius, assumed to coincide with the innermost stable circular orbit $R_{\mathrm{ISCO}} ; r_{\text {in }}$ is the apparent

\footnotetext{
${ }^{3}$ The most commonly used implementation of the disk-blackbody model is diskbb in XSPEC (Arnaud, 1996).
} 
inner radius obtained from spectral fitting with the diskblackbody approximation; $\alpha$ depends on the spin of the $\mathrm{BH}(\alpha=1$ for a Schwarzschild $\mathrm{BH}, \alpha=1 / 6$ for an extreme Kerr BH). The numerical factor $\xi$ was introduced (Kubota et al., 1998) to obtain a correctly normalized bolometric disk luminosity, taking into account that the fitted peak temperature occurs at $R=(49 / 36) R_{\text {in }}$ because of the notorque boundary conditions. With this approximation, and with the choice of $\kappa=1.7$ (Shimura and Takahara, 1995), the physical inner-disk radius is related to the fitted value by

$R_{\text {in }} \equiv\left(\xi^{1 / 2} \kappa\right)^{2} r_{\text {in }} \approx(1.09)^{2} r_{\text {in }}$.

An alternative estimate of $\xi \kappa^{2}$, which takes into account relativistic corrections, suggests that

$R_{\text {in }} \approx(1.35)^{2} r_{\text {in }}$

(Fabian et al., 2004), which is equivalent to a choice of $\kappa \approx 2.1$ for the hardening factor in (3). A hardening factor as high as $\kappa \approx 2.6$ was empirically estimated for some Galactic BHs in the high/soft state (Shrader and Titarchuk, 2003).

The bolometric luminosity can be derived from the observed flux

$L_{\text {disk }}=2 \pi d^{2} f_{\text {bol }}(\cos i)^{-1}$,

where $d$ is the source distance and $i$ the viewing angle (the disk flux being higher in the direction perpendicular to the disk plane). In addition, the disk luminosity can be directly related to the accretion rate, from general energy-conservation principles. Ignoring relativistic corrections,

$L_{\mathrm{disk}}=\frac{G M \dot{M}}{2 R_{\mathrm{in}}} \approx \frac{1}{12 \alpha} \dot{M} c^{2} \equiv \eta \dot{M} c^{2}$.

From (1), (2), and (6) we have

$$
\begin{aligned}
M & \approx \frac{c^{2} \xi \kappa^{2} \eta}{G(\sigma \pi)^{1 / 2}} L_{\text {disk }}^{1 / 2} T_{\text {in }}^{-2} \\
& \approx 10.0\left(\frac{\eta}{0.1}\right)\left(\frac{\xi \kappa^{2}}{1.19}\right)\left(\frac{L_{\text {disk }}}{5 \times 10^{38} \mathrm{erg} \mathrm{s}^{-1}}\right)^{1 / 2}\left(\frac{k T_{\text {in }}}{1 \mathrm{keV}}\right)^{-2} M_{\odot},
\end{aligned}
$$

which can also be expressed as $L_{\text {disk }} \sim M^{2} T_{\text {in }}^{4}$. This is the fundamental evolutionary track of an accretion disk in the thermal-dominant state. As their accretion rate varies, individual sources are expected to move along tracks of constant $M$ and $R_{\text {in }}$. This has been observationally verified for Galactic BHs (e.g., Kubota and Makishima, 2004; Miller et al., 2004), at least until their disk luminosity approaches the classical Eddington limit, $L_{\mathrm{Edd}} \approx 1.3 \times$ $10^{38}\left(M / M_{\odot}\right) \mathrm{erg} \mathrm{s}^{-1}$. Despite the various approximations, (7) works well (within a factor of 2) when applied to the masses of Galactic BHs, assuming radiative efficiencies $\lesssim 0.2$. Theoretical and observational arguments suggest that stellar-mass BHs are typically found in a thermaldominant state when their luminosity and corresponding accretion rate are $\sim 0.1-1$ times the classical spherical Eddington limit.

\section{Spectral arguments for intermediate-mass BHs}

\subsection{Soft X-ray emission in $U L X S$}

ULX X-ray spectra can also be modelled with a powerlaw plus a thermal component, much cooler than that observed from Galactic BHs - typically, with colour temperatures $k T_{\text {in }} \sim 0.12-0.20 \mathrm{keV}$ for the brightest sources (Miller et al., 2004; Feng and Kaaret, 2005; Stobbart et al., 2006). If (7) is applied, with total $0.3-10 \mathrm{keV}$ luminosities $\approx 1-3 \times 10^{40} \mathrm{erg} \mathrm{s}^{-1}$, the inferred masses are $\sim 1000-2000 M_{\odot}$, or a factor of two lower if we consider only the luminosity in the thermal component. This argument has been used in support of the intermediate-mass BH interpretation of ULXs (Miller et al., 2004).

However, it is unclear whether this argument can be directly applied to ULXs. There is still little direct evidence that the "soft excess" in ULXs is due to disk emission, partly because of our limited observing band. Various alternative interpretations have been suggested (e.g., Gonçalves and Soria, 2006; Stobbart et al., 2006; Ebisawa et al., 2003). For example, analogous soft-excesses in AGN, and particularly in narrow-line Seyfert 1 galaxies, can be explained as blurred, ionized absorption (mostly in the $\sim 0.5-2 \mathrm{keV}$ band) and reprocessing of the primary power-law-like spectrum in a fast outflow (Gierliński and Done, 2004; Chevallier et al., 2006). Gonçalves and Soria (2006) showed that a similar interpretation can be applied to at least some ULXs, whose X-ray spectra are very similar to those of narrow-line Seyferts. Downscattering of harder photons by a cooler outflow is another mechanism proposed to explain the soft excess (King and Pounds, 2003; Laming and Titarchuk, 2004). Even if we accept that the soft excess is indeed thermal emission from a disk (as we shall do in the rest of this paper), we need to keep in mind that some of the disk-blackbody scaling relations summarized in Section 1 have been confirmed observationally for the thermal-dominant state (Remillard and McClintock, 2006), but have to be modified when a consistent fraction of the accretion power is not directly radiated by the disk (Section 3).

\subsection{Cool disks and power-law component}

Two key issues need to be addressed by any ULX spectral model. The first is that the X-ray spectra of most ULXs are dominated by a broad, power-law-like component, at least in the limited energy range covered by Chandra and $X M M$-Newton. The thermal component represents only $\sim 10-30$ percent of the $0.3-10 \mathrm{keV}$ emission (Stobbart et al., 2006; Feng and Kaaret, 2005; Winter et al., 2006). Physically, this suggests that most of the accretion power is not directly radiated by the disk, but is efficiently transferred in other forms (mechanical, thermal or magnetic 
energy) to an upscattering medium, and then partly radiated with a non-thermal (power-law-like) spectrum. In Galactic stellar-mass BHs, this happens more commonly when the system is in the low/hard state, accreting at less than a few percent of their Eddington rate. But for transient sources, it also happens briefly near the peak of their outbursts, when they are probably accreting above their Eddington rate (very high state).

The second issue to be addressed is why the disk appears so cold for its luminosity. Even when we take into account only the luminosity in the fitted thermal component, ULX disks can radiate up to $\approx 10^{40} \mathrm{erg} \mathrm{s}^{-1}$ with a much lower peak temperature than that observed from Galactic BHs in their high state, even though the latter are an order of magnitude less luminous. A standard, truncated disk, replaced in the inner region by a radiatively-inefficient flow (Narayan and Yi, 1994), would produce a power-lawdominated spectrum with a cold disk component (low/hard state). However, if ULXs are in the low/hard state at luminosities $\approx 10^{40} \mathrm{erg} \mathrm{s}^{-1}$ (and hence, at mass accretion rates $\gtrsim 10^{6} M_{\odot} \mathrm{yr}^{-1}$ ), their $\mathrm{BH}$ masses should be $\gtrsim 10^{4} M_{\odot}$. The formation of such massive remnants in the local Universe is difficult to explain with existing models of stellar or star cluster evolution, and the existence of primordial intermediate-mass BHs is not yet supported by independent evidence. Therefore, we need to consider alternative scenarios, in which the accretion rate is high, but the disk is or appears cooler than a standard disk in a thermal-dominant state, for the same accretion rate and $\mathrm{BH}$ mass.

In the rest of this paper, we will discuss phenomenological corrections to the disk-blackbody equations, to estimate what physical effects may produce more luminous but cooler disks. We will also illustrate the effects that those corrections have on the $\mathrm{BH}$ mass estimates.

\section{Parametrical modifications of the disk-blackbody equations}

\subsection{Constant removal of power}

The first plausible scenario we consider is that a constant fraction of power $\epsilon>0$ is extracted from the disk at each radius (independent of radius); for example, to power a corona. The radiative flux from the disk becomes

$\sigma T_{\mathrm{eff}}(R)^{4} \approx(1-\epsilon) \frac{3 G M \dot{M}}{8 \pi R^{3}}$.

As a result, the peak temperature $T_{\text {in }} \rightarrow T_{\text {in }}^{\prime}=(1-\epsilon)^{1 / 4} T_{\text {in }}$, and $L_{\text {disk }} \rightarrow L_{\text {disk }}^{\prime}=(1-\epsilon) L_{\text {disk }}$. Neither $R_{\text {in }}$ nor $r_{\text {in }}$ changes. The emitted spectrum is still a disk-blackbody. As $\epsilon$ increases, the disk becomes cooler and less luminous, for a fixed accretion rate, and its parameters move downwards along the same $L_{\text {disk }} \sim M^{2} T_{\text {in }}^{4}$ track. This scenario is equivalent to changing the effective accretion rate $\dot{M} \rightarrow \dot{M}^{\prime}=(1-\epsilon) \dot{M}$. It follows from the previous argument that the BH mass is still given by (7), independent of $\epsilon$. Hence, removal of a constant fraction of accretion power does make the disk cooler but does not explain the high $\mathrm{BH}$ masses inferred from ULX spectral fitting.

\subsection{Hardening of the disk photons}

Another simple modification to the standard-disk scenario is to assume that an increasing fraction of the emitted disk photons are upscattered by relativistic electrons at the disk photosphere. This is equivalent to increasing the hardening factor $\kappa$, typically from $\approx 1.7$ to $\approx 2.5$ (Davis et al., 2005 , 2006). Observationally, this spectral hardening occurs at accretion rates near or just above Eddington.

In the ( $\left.T_{\text {in }}, L_{\text {disk }}\right)$ plane, the effect is to move the source horizontally to the right: higher colour temperature $T_{\text {in }}$ but same effective temperature $T_{\mathrm{in}} / \kappa$ and same luminosity $L_{\text {disk }}$. The true inner-disk radius $R_{\text {in }}$ does not change, while the apparent disk radius decreases: $r_{\text {in }} \rightarrow r_{\text {in }}^{\prime}=[\kappa /$ $(\kappa+\Delta \kappa)]^{2} r_{\text {in. }}$. This does not affect the mass estimate, if one has properly taken into account its dependence on $T_{\text {in }} / \kappa$, as we did in (7).

\subsection{Partial covering of the disk}

The third effect that needs to be taken into account is a partial covering of the disk surface: a fraction $X$ of photons may be absorbed or more generally removed from the diskblackbody spectrum by clouds or a moderately opticallythick corona. They are re-emitted in other spectral bands or components (e.g., X-ray power-law, or infrared). In a self-consistent analysis, one must of course account for all upscattering, downscattering and absorption effects, redistributing the total available accretion power into the various components. But for the purpose of relating the disk emission parameters to the $\mathrm{BH}$ mass, we can simply consider those photons as being lost from the disk spectrum. The effect is to reduce the observed disk flux and consequently the inferred luminosity: $L_{\text {disk }} \rightarrow L_{\text {disk }}^{\prime}=$ $(1-X) L_{\text {disk }}$. The spectral shape is not altered. The peak colour temperature $T_{\text {in }}$ is not changed. $R_{\text {in }}$ stays the same but the apparent disk radius decreases: $r_{\text {in }} \rightarrow r_{\text {in }}^{\prime}=$ $(1-X)^{1 / 2} r_{\text {in }}$, because $r_{\text {in }}$ is indirectly derived from the flux normalization. The $\mathrm{BH}$ mass inferred from the observed luminosity and temperature via (7) is a factor $(1-X)^{1 / 2}$ less than the true mass.

The combined effect of the modifications described in Sections 3.1, 3.2, and 3.3 is to move the location of the source to the right-hand-side of its thermal track, in the $\left(T_{\text {in }}, L_{\text {disk }}\right)$ plane (Fig. 1). The precise displacement depends on the relative importance of the parameters $\epsilon, \Delta \kappa$ and $X$. Physically, it leads to lower estimates for the radius $r_{\text {in }}$ (sometimes much lower than the innermost stable orbit), and may lead to an underestimate of the BH mass if not properly accounted for. These empirical corrections may be used to explain the very high state (or steep-power-law state) of accreting black holes (Remillard and McClintock, 2006). They do not help explain the high masses and large radii inferred for ULXs. 


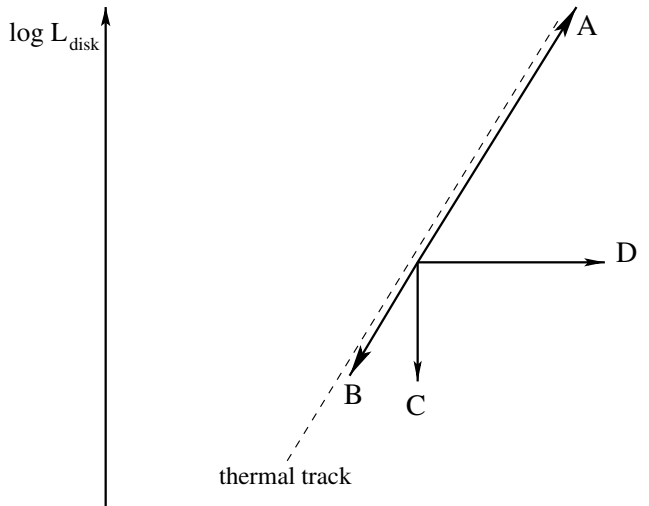

$\log \mathrm{T}_{\text {in }}$

Fig. 1. Evolution of the source parameters in the $\left(T_{\text {in }}, L_{\text {disk }}\right)$ plane, due to various competing effects at high accretion rates. Dashed line: thermal track, characterized by $L_{\text {disk }} \sim T_{\text {in }}^{4} M^{2}$; A: effect of increasing the accretion rate; $\mathrm{B}$ : effect of injecting some of the accretion power into a corona or outflow (uniformly at all radii); $\mathrm{C}$ : effect of removing some of the disk photons via absorption or upscattering into the power-law component; $\mathrm{D}$ : effect of spectral hardening due to electron scattering near the disk photosphere (equivalent to increasing the colour temperature without changing the effective temperature).

\section{A two-zone structure of the inflow}

For high accretion rates, it is plausible to speculate that accretion power may not be uniformly removed from the whole disk (Section 3.1) and/or disk photons may not be uniformly "lost" independent of radius. More likely, those losses may be strongly concentrated in the inner-disk region (Done and Kubota, 2006; Soria and Kuncic, submitted for publication). For example, the inner disk may be disrupted or simply occulted by an optically-thick outflow. Or, all of its photons may be comptonized and emerge as a power-law component. Alternatively, the inner disk may still be present and visible, but most of its accretion power is extracted via non-radiative processes, such as mechanical outflows or Poynting flux (Kuncic and Bicknell, 2004). Some of the power released through those channels is then converted to non-thermal photons.

Hence, a possible phenomenological way to correct the standard disk equations is to assume the presence of a transition radius $R_{\mathrm{c}} \gg R_{\mathrm{ISCO}}$. For $R>R_{\mathrm{c}}$, the inflow can be approximated by a standard disk, such that

$L_{\text {disk }} \approx 4 \pi \sigma T_{\mathrm{c}}^{4} R_{\mathrm{c}}^{2}$,

where $T_{\mathrm{c}} \equiv T\left(R_{\mathrm{c}}\right)$ is the maximum (observable) disk temperature. For $R_{\mathrm{ISCO}}<R<R_{\mathrm{c}}$, we assume that all the accretion power comes out in the power-law-like component, and the standard disk either is not directly visible or gives a negligible direct contribution.

Regardless of the details, in this scenario, (2) no longer holds or is no longer relevant to the observed spectrum. It is replaced by

$R_{\mathrm{c}}=F R_{\mathrm{ISCO}}$ with $F=F(\dot{M})>1$. The visible disk radiates only the accretion power released from the outer radius to $R_{\mathrm{c}}$. The effective radiative efficiency of that part of the inflow $\sim M / R_{\mathrm{c}} \sim 1 /(12 \alpha F)<0.1$. Therefore, we have (cf. (6) and (7))

$$
\begin{aligned}
L_{\mathrm{disk}} & \approx \frac{G M \dot{M}}{2 R_{\mathrm{c}}} \approx \frac{1}{12 \alpha F} \dot{M} c^{2} \equiv \frac{\eta}{F} \dot{M} c^{2} \\
M & \approx \frac{10.0}{F}\left(\frac{\eta}{0.1}\right)\left(\frac{\xi \kappa^{2}}{1.19}\right)\left(\frac{L_{\mathrm{disk}}}{5 \times 10^{38} \mathrm{erg} \mathrm{s}^{-1}}\right)^{1 / 2}\left(\frac{k T_{\mathrm{in}}}{1 \mathrm{keV}}\right)^{-2} M_{\odot} \\
& \approx \frac{790}{F}\left(\frac{\eta}{0.1}\right)\left(\frac{\xi \kappa^{2}}{1.19}\right)\left(\frac{L_{\mathrm{disk}}}{5 \times 10^{39} \mathrm{erg} \mathrm{s}^{-1}}\right)^{1 / 2}\left(\frac{k T_{\text {in }}}{0.2 \mathrm{keV}}\right)^{-2} M_{\odot},
\end{aligned}
$$

where $L_{\text {disk }} \sim 5 \times 10^{39} \mathrm{erg} \mathrm{s}^{-1}$ and $k T_{\text {in }} \sim 0.2 \mathrm{keV}$ are typical values found in ULXs. We speculate that the high masses inferred for some of those systems are not evidence of intermediate-mass BHs, but are simply a consequence of a high value $F \gtrsim 10$ not properly taken into account in the disk modelling. Defining a dimensionless accretion rate

$\dot{m} \equiv \dot{M} / \dot{M}_{\text {Edd }} \equiv \frac{0.1 \dot{M} c^{2}}{L_{\text {Edd }}}$,

the bolometric disk luminosity integrated for $R>R_{\mathrm{c}}$ can more usefully be expressed as

$L_{\mathrm{disk}} \approx\left(\frac{\dot{m}}{F}\right)\left(\frac{\eta}{0.1}\right) L_{\mathrm{Edd}}$.

\section{Observational predictions}

It was suggested that the disk structure parameterized in Section 4 is applicable to sources with very high accretion rates, $\dot{m}>1$ (Done and Kubota, 2006; Soria and Kuncic, submitted for publication). To estimate the $\mathrm{BH}$ mass and accretion rate of a source in that state, from the observed disk luminosity and temperature, we need to estimate $F=R_{\mathrm{c}} / R_{\mathrm{ISCO}}$, either by measuring it directly, or by understanding how it depends on $\dot{m}$. The simplest way to estimate $F$, at least as an order of magnitude, is to recall that in this scenario, all of the accretion power released at $R>R_{\mathrm{c}}$ is radiated by the disk, and some or most of the power released at $R_{\mathrm{ISCO}}<R<R_{\mathrm{c}}$ is eventually radiated as power-law photons, without contributing to the thermal disk component. Since the accretion power liberated down to a radius $R$ scales $\sim 1 / R$, one can estimate that $F \gtrsim L_{\text {tot }} /$ $L_{\text {disk. }}$. The inequality sign takes into account the fact that the radiative efficiency of the non-thermal processes in the inner region is less than the efficiency of blackbody emission in a standard disk. For the most luminous nearby ULXs, extrapolating from the relative fluxes of the powerlaw and thermal components in the $0.3-10 \mathrm{keV}$ band, the luminosity ratio $L_{\text {tot }} / L_{\text {disk }} \sim 3-20$; in a few other, more distant ULXs, there is only a lower limit $\gtrsim 10$ for this ratio. Hence, we infer that $F \gtrsim 10$ for typical ULXs.

From the fitted luminosity and temperature of the disk (9), typical values of $R_{\mathrm{c}} \gtrsim 5000 \mathrm{~km}$ are found for many 
ULXs, i.e., $\sim 100$ times larger than typical inner-disk radii in stellar-mass $\mathrm{BH}$. If $F \gtrsim 10$, it means that $\mathrm{BH}$ masses in those ULXs are only required to be $\$ 10$ times larger than typical BH masses in Galactic systems. This simple argument suggests that ULX masses are $\lesssim 100 M_{\odot}$. See Soria and Kuncic (submitted for publication) for further investigation of this issue.

The location or evolutionary track of a source in the $\left(T_{\mathrm{c}}, L_{\mathrm{disk}}\right)$ plane provides another observational constraint. To predict the displacement of a source from its thermal track when $F>1$, we need to understand how $F$ and $T_{\mathrm{c}}$ vary as a function of $\dot{m}$. For a fixed BH mass, the radiative flux equation of a standard disk tells us that $T_{\mathrm{c}} \sim R_{\mathrm{c}}^{-3 / 4} \dot{m}^{1 / 4}$. It was suggested (Poutanen et al., 2007; Begelman et al., 2006; Soria and Kuncic, submitted for publication) that $R_{\mathrm{c}} \sim \dot{m}$, based on plausible physical processes that may form such a transition radius. If so, from (9) we expect that, as the accretion rate $\dot{m}$ increases, a source will move along a track defined by $L_{\text {disk }} \approx$ constant, $T_{\mathrm{c}} \sim \dot{m}^{-1 / 2}$ (Fig. 2).

The luminosity track is very sensitive to the radial temperature distribution on the disk, at $R>R_{\mathrm{c}}$. Disk models with a distribution flatter than $R^{-3 / 4}$ are sometimes used. For example, it was found (Kubota et al., 2005) that $T \sim R^{-0.7}$ may provide a more accurate fit to the X-ray spectral data of Galactic BHs. In that case, we expect $L_{\text {disk }} \sim T_{\mathrm{c}}^{-0.44}$, with $T_{\mathrm{c}} \sim \dot{m}^{-0.45}$. For a disk dominated by $\mathrm{X}$-ray irradiation (as may be the case if $R \gg R_{\mathrm{ISCO}}$ ), $T \sim R^{-0.5}$. In that case, $L_{\text {disk }} \sim T_{\mathrm{c}}^{-4}$, with $T_{\mathrm{c}} \sim \dot{m}^{-0.25}$. See Soria and Kuncic (submitted for publication) for further discussions and a comparison with observations.

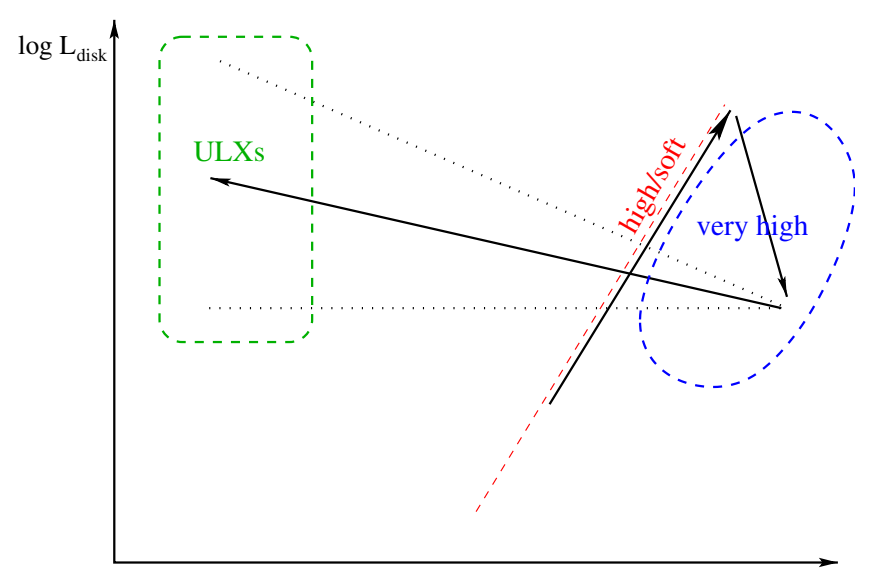

$\log \mathrm{T}_{\text {in }}$

Fig. 2. Suggested X-ray spectral evolution of $\mathrm{BH}$ accretion disks for accretion rates $0.1 \lesssim \dot{m} \lesssim 20$. Sources first move up the thermal track, until their disk luminosity $L_{\text {disk }} \approx L_{\text {Edd }}$ (high/soft state). Then, they move to the very high state (or steep-power-law state), generally characterized by a higher peak temperature, lower disk luminosity and a dominant, steep-power-law component. Finally, as $\dot{m}$ increases further, they may move to the ULX region, characterized by a large transition radius between outer disk and inner non-thermal region, and a dominant, flatter power-law component. The location of the thermal track (high/soft state) is determined by the $\mathrm{BH}$ mass; the horizontal shift towards lower apparent temperatures is mostly determined by the accretion rate.
Regardless of the precise functional forms of $F(\dot{m})$ and $T_{\mathrm{c}}\left(R_{\mathrm{c}}\right)$, the significant result is that we expect a source to move to the left-hand-side of its thermal track, as the accretion rate increases. Along that track, the luminosity of the disk component stays constant or increases, even if the observed peak temperature decreases. We speculate that the effects described in Sections 3.1, 3.2 and 3.3 dominate when $\dot{m} \gtrsim 1$, and the shift to larger transition radii and lower peak temperatures becomes the dominant term for $\dot{m} \gg 1$. Thus, we predict that an accreting BH may initially move up along its thermal track, then slightly down and to the right, then cross the thermal track and move to the left (Fig. 2). As it does so, we also expect the source to become more and more dominated by non-thermal emission components.

The fitted X-ray properties of many bright ULXs are qualitatively consistent with this phenomenological scenario. Their disks are very luminous but have a low peak temperature. The total power output is dominated by a power-law component, perhaps in addition to other nonthermal and non-radiative mechanisms (e.g., outflows). Their characteristic variability timescales are consistent with large disk radii (Soria and Kuncic, submitted for publication). For example, let us consider an accreting $\mathrm{BH}$ with $M \approx 50 M_{\odot}$, and a disk luminosity $\approx 5 \times 10^{39} \mathrm{erg} \mathrm{s}^{-1}$ $\approx L_{\text {Edd }}$. If $\dot{m} \approx 1$ and $F=1$, we expect its spectrum to be dominated by the disk component, with a peak temperature $\approx 0.8 \mathrm{keV}$ and a characteristic size of the X-rayemitting region $\sim 6 G M / c^{2} \sim 500 \mathrm{~km}$. Although the luminosity is consistent with an average ULX, its spectral properties are not. However, let us allow for $\dot{m} \approx 20$, and consequently $F \approx 20$. In that case, we expect that the source will still have $L_{\text {disk }} \approx L_{\mathrm{Edd}} \approx 5 \times 10^{39} \mathrm{erg} \mathrm{s}^{-1}$, but a peak temperature $\approx 0.8 \times 20^{-0.5} \mathrm{keV} \approx 0.18 \mathrm{keV}$, a characteristic radius $\sim 20 \times 6 G M / c^{2} \sim 10,000 \mathrm{~km}$, and a dominant $\mathrm{X}$-ray power-law component with a luminosity $\gtrsim 5 \times$ $10^{39} \mathrm{erg} \mathrm{s}^{-1}$. These are more typical ULX parameters. If we had observed such a source, determining only its fitted values of $L_{\text {disk }}$ and $T_{\mathrm{c}}$, and inserting those values directly into (7) instead (9), we would have incorrectly interpreted the source as an intermediate-mass $\mathrm{BH}$ with $M \approx 1000 M_{\odot}$.

\section{Conclusions}

It is now recognized that it may not be possible to estimate $\mathrm{BH}$ masses in ULXs using the standard disk-blackbody model. If we accept that the soft excess is the disk emission component (which at this stage is only one possible hypothesis), we need to account for the fact that ULX spectra are power-law dominated and their thermal component has a remarkably low peak temperature. We briefly reviewed simple phenomenological corrections to the standard disk model, which have been used to explain the very high state of Galactic BHs. However, they do not help explaining the ULX spectral properties. We then discussed the effects of introducing a transition radius $R_{\mathrm{c}} \gg 6 \mathrm{GM} / \mathrm{c}^{2}$, 
such that for $R>R_{\mathrm{c}}$ the accretion flow is a standard disk, while for $R<R_{\mathrm{c}}$ the disk is either drained of all its power via non-thermal processes, or its photons are completely reprocessed by an optically-thick comptonizing medium. In this case, the peak disk emission comes from $R \approx R_{\mathrm{c}}$, while the inner part of the inflow emits with a power-lawlike spectrum.

We speculate that $R_{\mathrm{c}} / R_{\mathrm{ISCO}} \sim \dot{m}$, where $\dot{m}$ is the accretion rate in Eddington units. With this parameterization, for a fixed $\mathrm{BH}$ mass, the peak colour temperature $T_{\mathrm{c}} \sim \dot{m}^{p}$ with $p \approx-0.5$, and $L_{\mathrm{disk}} \sim T_{\mathrm{c}}^{q}$, with $q \leqslant 0$. The predicted effect is to move the source to the left-hand-side of its thermal track in the $\left(T_{\mathrm{c}}, L_{\mathrm{disk}}\right)$ plane. This behaviour may be incorrectly read as standard disk emission from a more massive $\mathrm{BH}$. We argue that this had led to the flawed intermediate-mass $\mathrm{BH}$ interpretation. In our scenario, if the accretion rate $\dot{m} \sim 20$, the $\mathrm{BH}$ mass can be $\sim 50$ $100 M_{\odot}$; hence, perhaps still consistent with their formation via stellar processes, in very special circumstances. On the other hand, the BH mass may not be much smaller than $\sim 50 M_{\odot}$, if we assume that $L_{\text {disk }} \lesssim L_{\text {Edd }}$ and $L_{\text {tot }} \lesssim$ a few $L_{\text {Edd. }}$ In this scenario, the peak flux contribution from the disk may come from $\sim 100$ gravitational radii. The dependence of the transition radius on the accretion rate needs to be taken into account also when scaling the frequency of X-ray quasi-periodic oscillations between sources of different mass. Lower frequencies may be caused by either an increase of $\dot{m}$ or an increase of $M$.

In summary, we speculate that ULXs may be defined as accreting BHs with masses a few times higher than Galactic $\mathrm{BHs}$, and persistent accretion rates at least an order of magnitude above their Eddington limit. None of them has been seen to move along its thermal track, typical of accretion rates $\sim 0.1-1$ Eddington. Perhaps this is due to the nature of their massive Roche-lobe-filling donor stars, which provide a consistently higher mass transfer rate. A physical interpretation of the transition radius, as well as a more detailed comparison with observed X-ray spectral and timing properties of some Galactic BHs and ULXs will be presented elsewhere (Soria and Kuncic, submitted for publication).

\section{Acknowledgements}

We thank G.V. Bicknell, A.C. Gonçalves, and J.E. McClintock for useful discussions and suggestions.

\section{References}

Arnaud, K.A. Astronomical data analysis software and systems V, in: Jacoby, G., Barnes, J. (Eds.), ASP Conf. Series, vol. 101, p. 17, 1996.

Begelman, M.C., King, A.R., Pringle, J.E. The nature of SS433 and the ultraluminous X-ray sources. MNRAS 370, 399, 2006.
Chevallier, L., Collin, S., Dumont, A.-M., Czerny, B., Mouchet, M., Gonçalves, A.C., Goosmann, R. The role of absorption and reflection in the soft X-ray excess of Active Galactic Nuclei. I. Preliminary results. A\&A 449, 493, 2006.

Davis, S.W., Done, C., Blaes, O.M. Testing accretion disk theory in black hole X-ray binaries. ApJ 647, 525, 2006.

Davis, S.W., Blaes, O.M., Hubeny, I., Turner, N.J. Relativistic accretion disk models of high-state black hole X-ray binary spectra. ApJ 621, 372, 2005

Done, C., Kubota, A. Disc-corona energetics in the very high state of Galactic black holes. MNRAS 371, 1216, 2006.

Ebisawa, K., Życki, P., Kubota, A., Mizuno, T., Watarai, K. Accretion disk spectra of ultraluminous X-ray sources in nearby spiral galaxies and Galactic superluminal jet sources. ApJ 597, 780, 2003.

Fabian, A.C., Ross, R.R., Miller, J.M. On the observed disc temperatures of accreting intermediate-mass black holes. MNRAS 355, 359, 2004.

Feng, H., Kaaret, P. XMM-Newton observations of ultraluminous X-ray sources in nearby galaxies. ApJ 633, 1052, 2005.

Gierliński, M., Done, C. Is the soft excess in active galactic nuclei real? MNRAS 349, L7, 2004.

Gonçalves, A.C., Soria, R. On the weakness of disc models in bright ULXs. MNRAS 371, 673, 2006.

King, A.R., Pounds, K.A. Black hole winds. MNRAS 345, 657, 2003.

Kubota, A., Tanaka, Y., Makishima, K., Ueda, Y., Dotani, T., Inoue, H., Yamaoka, K. Evidence for a black hole in the X-ray transient GRS 1009-45. PASJ 50, 667, 1998.

Kubota, A., Ebisawa, K., Makishima, K., Nakazawa, K. On the temperature profile of radiatively efficient geometrically thin disks in black hole binaries with the ASCA GIS. ApJ 631, 1062, 2005.

Kubota, A., Makishima, K. The three spectral regimes found in the stellar black hole XTE J1550-564 in its high/soft state. ApJ 601, 428, 2004.

Kuncic, Z., Bicknell, G.V. Dynamics and energetics of turbulent, magnetized disk accretion around black holes: a first-principles approach to disk-corona-outflow coupling. ApJ 616, 669, 2004.

Laming, J.M., Titarchuk, L. Outflows near an accreting black hole: ionization and temperature structures. 615, L121, 2004.

Makishima, K., Maejima, Y., Mitsuda, K., Bradt, H.V., Remillard, R.A., Tuohy, I.R., Hoshi, R., Nakagawa, M. Simultaneous X-ray and optical observations of GX 339-4 in an X-ray high state. ApJ 308, 635, 1986.

Makishima, K., Kubota, A., Mizuno, T., et al. The nature of ultraluminous compact X-ray sources in nearby spiral galaxies. ApJ 535, 632, 2000.

Miller, J.M., Fabian, A.C., Miller, M.C. A comparison of intermediatemass black hole candidate ultraluminous X-ray sources and stellarmass black holes. ApJ 614, L117, 2004.

Narayan, R., Yi, I. Advection-dominated accretion: a self-similar solution. ApJ 428, L13, 1994.

Poutanen, J., Lipunova, G., Fabrika, S., Butkevich, A.G., Abolmasov, P. Super-critically accreting stellar-mass black holes as ultraluminous Xray sources. MNRAS 377, 1187, 2007.

Remillard, R.A., McClintock, J.E. X-ray properties of black-hole binaries. ARA\&A 44, 49, 2006.

Shimura, T., Takahara, F. On the spectral hardening factor of the X-ray emission from accretion disks in black hole candidates. ApJ 445, 780, 1995.

Shrader, C.R., Titarchuk, L. A method for black hole mass determination in accretion-powered X-ray sources. ApJ 598, 168, 2003.

Soria, R., Kuncic, Z. MNRAS, submitted for publication.

Stobbart, A.-M., Roberts, T.P., Wilms, J. XMM-Newton observations of the brightest ultraluminous X-ray sources. MNRAS 368, 397, 2006.

Winter, L.M., Mushotzky, R.F., Reynolds, C.S. XMM-Newton archival study of the ultraluminous X-ray population in nearby galaxies. ApJ 649, 730, 2006. 